\title{
Mitral valve replacement in children Comparative study of pre- and postoperative haemodynamics and left ventricular function
}

\author{
E G BENMIMOUN, B FRIEDLI, W RUTISHAUSER, B FAIDUTTI \\ From Clinique de Pédiatrie, Centre de Cardiologie, and Clinique de Chirurgie Cardiovasculaire, Hôpital Cantonal \\ Universitaire, Geneva, Switzerland
}

SUMMARY Haemodynamic variables and left ventricular function were studied before and after mitral valve replacement in 44 children age 3 to 17 years (mean 11.9 years). Thirty-nine StarrEdwards prostheses and five Hancock prostheses were used; postoperative study took place two to six months (mean 3.9 months) after operation. Pulmonary hypertension was present preoperatively in most patients, with mean pulmonary artery pressures of 18 to 75 (mean $46.5 \mathrm{mmHg}$ ). Postoperatively there was a pronounced drop in pressure to a mean value of $25.6 \mathrm{mmHg}$, partially explained by a decrease in pulmonary capillary wedge pressure. Pulmonary arteriolar resistance, however, also decreased conspicuously from an average of 590 dynes $\mathrm{s} \mathrm{cm}^{-5} \mathrm{~m}^{-2}$ preoperatively to 282 dynes s $\mathrm{cm}^{-5} \mathrm{~m}^{-2}$ postoperatively. A return to normal resistance was seen in every case when preoperative resistance did not exceed 650 dynes $\mathrm{s} \mathrm{cm}^{-5} \mathrm{~m}^{-2}$; above this threshold some degree of pulmonary hypertension often persisted. The residual gradient across the prosthetic valve was slightly higher for the Hancock than for the Starr-Edwards prosthesis (mean $8.7 \mathrm{mmHg}$, vs mean $6.9 \mathrm{mmHg}$ ).

The left ventricular end-diastolic volume was much increased before surgery, with a mean value of $190 \mathrm{ml} / \mathrm{m}^{2}$; it decreased conspicuously after operation to $103 \mathrm{ml} / \mathrm{m}^{2}$. The left ventricular ejection fraction ranged from $40 \%$ to $76 \%$ (mean $57 \%$ ) before operation; there was no significant change after operation, with values ranging from $40 \%$ to $73 \%$.

This left ventricular dysfunction is probably the result of myocardial injury caused by a chronic volume overload and the sequelae of rheumatic carditis.

Mitral valve disease with regurgitation and stenosis gives rise to various haemodynamic consequences. First, there is left ventricular volume overload and dilatation of the left atrium and ventricle. Longstanding left ventricular volume overload may compromise left ventricular function. ${ }^{12}$ If the mitral valve disease is of rheumatic origin, left ventricular function may also be depressed as a consequence of myocardial fibrosis, secondary to myocarditis. Secondly, pulmonary venous congestion will result in pulmonary hypertension; with time, pulmonary vascular disease may develop, compromising the return to normal pressures after mitral valve function is restored. Severe mitral valve disease often requires valve replacement, which is usually reserved for very disabled patients. Various degrees of left ventricular dys-

Accepted for publication 13 April 1982 function and of pulmonary hypertension are usually therefore present, and the reversibility of such changes is uncertain. Haemodynamic assessment after mitral valve replacement is of use and has often been performed in adult patients, ${ }^{3-5}$ but very few data are available in children. ${ }^{6}$ We did not find any data concerning left ventricular function and volume after valve replacement in children.

We would like to report the results of postoperative catheterisation in 44 children who have undergone valve replacement for severe mitral valve disease, including the results of left ventricular volume studies in 30 children.

\section{Patients and methods}

Forty-four children ( 23 boys and 21 girls) with clinical and haemodynamic evidence of severe mitral valve 
disease were studied. The valve lesion was the result of rheumatic heart disease in 35 , and of congenital malformation in nine. Their ages ranged from 3 to 17 years (mean 11.9 years). Twenty-nine patients had mitral regurgitation associated with mitral stenosis; 15 patients had pure mitral regurgitation. In 30 patients, there was significant disease of another valve: 12 had aortic regurgitation, 12 had tricuspid regurgitation, six had aortic and tricuspid regurgitation. Two had a ventricular septal defect. Thirty-three patients were in grade IV heart failure (according to the New York Heart Association), nine in grade III, and two in grade II. Thirty-nine underwent mitral valve replacement with a Starr-Edwards prosthesis and five with a Hancock prosthesis; nine patients also had a tricuspid annuloplasty (De Vega), and two aortic valve replacement (one Björk and one Starr-Edwards prosthesis). Two patients had closure of a ventricular septal defect.

These 44 patients are part of a group of 171 children who underwent valve replacement from 1969 to 1980 in Geneva. ${ }^{7}$ Postoperative catheterisation was performed either to assess pulmonary pressure (when preoperative values were raised) or to rule out valve dysfunction in the presence of a systolic or diastolic murmur.

Cardiac catheterisation was performed two to six months (mean 3.9 months) after operation. The procedure was done under light sedation with a mixture of pethidine, promethazine, and chlorpromazine.
Routine cardiac catheterisation was performed by percutaneous puncture of the right femoral artery and vein. Cardiac output was measured by the Fick method, with oxygen consumption assumed (table of La Farge and Miettinen). Pulmonary vascular resistance was calculated by the following formulae: TPVR $=$ PA $\times 80 / \mathrm{CI}$; PAR $=(\mathrm{PA}-\mathrm{PAW}) \times 80 / \mathrm{CI}$, where TPVR is the total pulmonary vascular resistance in dynes $\mathrm{s} \mathrm{cm}^{-5} \mathrm{~m}^{-2}$, PAR is the pulmonary arteriolar resistance, PA and PAW are the mean pulmonary arterial and pulmonary arterial wedge pressures ( $\mathrm{mmHg}$ ), respectively, and $\mathrm{CI}$ is the cardiac index (litres/min per $\mathrm{m}^{2}$ ).

Thirty patients underwent left ventricular cineangiography in the right anterior oblique projection using 1 to $1.5 \mathrm{ml} / \mathrm{kg}$ of $76 \%$ Urografin. Ventricular volumes were calculated using the Sandler and Dodge formula $^{8}$ from planimetered surfaces on the right anterior oblique cineangiogram. Ejection fraction (EF) was calculated with the following formula: $\mathrm{EF} \%=(\mathrm{EDV}-\mathrm{ESV}) / \mathrm{EDV} \times 100$, where $\mathrm{EDV}$ and $\mathrm{ESV}$ are end-diastolic and end-systolic volumes, respectively. We took cardiac cycles early after opacification to obtain physiological information from cineangiocardiography. 9 The pre- and postextrasystolic systoles were excluded. Mean frequency systoles were taken when the patient was in atrial fibrillation. Absolute and indexed volume is reported in 27 patients only because the magnification factor was unknown in three.

able Haemodynamics and left ventricular function data

\begin{tabular}{|c|c|c|c|c|c|c|c|c|c|c|c|c|c|c|c|}
\hline $\begin{array}{l}\text { iase } \\
\text { lo. }\end{array}$ & Sex & Age & & $P A$ & $P A W$ & $M G$ & CI & $T P V R$ & $P A R$ & $N Y H A$ & $R M R$ & $\begin{array}{l}\text { Surgical } \\
\text { treatment }\end{array}$ & $L V E D$ & $E F$ & 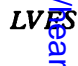 \\
\hline 1 & $\mathbf{F}$ & 15 & $\begin{array}{l}\text { Preop } \\
\text { Postop }\end{array}$ & $\begin{array}{ll}45 / 24 & \overline{33} \\
35 / 18 & \frac{25}{3}\end{array}$ & $\begin{array}{l}21 \\
15\end{array}$ & $\begin{array}{r}20 \\
8\end{array}$ & $\begin{array}{l}2.9 \\
4.0\end{array}$ & $\begin{array}{l}910 \\
500\end{array}$ & $\begin{array}{l}333 \\
200\end{array}$ & IV & - & $\begin{array}{l}\text { Starr } M \\
\text { An. } T\end{array}$ & $\begin{array}{l}80 \\
50\end{array}$ & $\begin{array}{l}60 \\
56\end{array}$ & 310 \\
\hline 2 & $\mathbf{M}$ & 15 & Preop & $90 / 50 \frac{68}{30}$ & 30 & $\overline{0}$ & 2.5 & 2190 & 1220 & IV & & Starr $M$ & - & - & $-\dot{0}$ \\
\hline 3 & $\mathbf{M}$ & 13 & Preop & $40 / 20 \frac{20}{28}$ & 20 & $\begin{array}{r}8 \\
25\end{array}$ & $\begin{array}{l}4 \cdot 9 \\
4 \cdot 4\end{array}$ & $\begin{array}{l}330 \\
510\end{array}$ & $\begin{array}{l}212 \\
150\end{array}$ & IV & - & Starr $\mathbf{M}$ & $\overline{379}$ & $\overline{61}$ & 147을 \\
\hline & & & Postop & $28 / 11 \overline{16}$ & 13 & 7 & 4.0 & 320 & 60 & $\bar{I}$ & - & & 189 & 53 & \\
\hline 4 & $\mathbf{F}$ & 11 & $\begin{array}{l}\text { Preop } \\
\text { Postop }\end{array}$ & $\begin{array}{l}65 / 45 \overline{55} \\
36 / 10 . \overline{17}\end{array}$ & $\begin{array}{r}30 \\
6\end{array}$ & $-\frac{}{5}$ & $\begin{array}{l}1.9 \\
3.6\end{array}$ & $\begin{array}{r}2380 \\
350\end{array}$ & $\begin{array}{r}1050 \\
240\end{array}$ & IV & - & Starr $\mathbf{M}$ & - & - & - \\
\hline 5 & $\mathbf{F}$ & 13 & Preop & $42 / 13 \frac{1}{34}$ & 28 & 17 & 2.4 & 1130 & 200 & IV & & Starr $\mathbf{M}$ & $\overline{176}$ & $\overline{62}$ & $67 \frac{1}{0}$ \\
\hline & & & Postop & $26 / 3 \overline{16}$ & 8 & 7 & $4 \cdot 1$ & 310 & 160 & I-II & - & & 109 & 50 & \\
\hline 6 & $\mathbf{M}$ & 7 & Preop & $30 / 10 \quad \overline{24}$ & 13 & $\overline{6}$ & $4 \cdot 0$ & 480 & 220 & III & & Hancock M & 一 & - & $-N$ \\
\hline 7 & $\mathbf{F}$ & 13 & Preop & $105 / 58 \frac{11}{75}$ & 45 & 44 & $\begin{array}{l}4.0 \\
1.9\end{array}$ & $\begin{array}{r}290 \\
3160\end{array}$ & $\begin{array}{r}150 \\
1260\end{array}$ & IV & - & Starr $\mathbf{M}$ & $\overline{337}$ & 65 & $\overline{120}$ \\
\hline & & & Postop & $40 / 14 \overline{26}$ & 5 & 2 & 40 & 520 & 420 & I-II & - & & 119 & 65 & 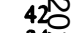 \\
\hline 8 & $\mathbf{F}$ & 16 & Preop & $\begin{array}{l}58 / 29 \quad 38 \\
30 / 12\end{array}$ & $\begin{array}{l}28 \\
11\end{array}$ & $\begin{array}{l}22 \\
10\end{array}$ & $\begin{array}{l}2.5 \\
3.4\end{array}$ & $\begin{array}{r}1220 \\
470\end{array}$ & $\begin{array}{l}920 \\
210\end{array}$ & IV & - & Starr $\mathbf{M}$ & $\begin{array}{l}95 \\
59\end{array}$ & $\begin{array}{l}64 \\
52\end{array}$ & \\
\hline 9 & $\mathbf{M}$ & 17 & Preop & $80 / 40 \frac{60}{50}$ & 29 & 24 & 2.7 & 1780 & 920 & IV & & Starr $\mathbf{M}$ & 116 & 54 & 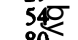 \\
\hline 0 & $\mathbf{F}$ & 16 & Postop & $\begin{array}{l}75 / 40 \leq 50 \\
58 / 25\end{array}$ & $\begin{array}{l}15 \\
30\end{array}$ & 8 & $\begin{array}{l}2 \cdot 1 \\
2 \cdot 2\end{array}$ & $\begin{array}{l}1900 \\
1450\end{array}$ & $\begin{array}{r}1330 \\
360\end{array}$ & II & $t$ & Starr $M$ & $\begin{array}{l}136 \\
196\end{array}$ & $\begin{array}{l}41 \\
76\end{array}$ & 46 \\
\hline 0 & $\boldsymbol{F}$ & 10 & $\begin{array}{l}\text { Preop } \\
\text { Postop }\end{array}$ & $25 / 14 \quad \frac{40}{18}$ & 11 & 8 & $\begin{array}{l}2.2 \\
2.5\end{array}$ & 580 & 220 & I-II & - & & 85 & 48 & \\
\hline .1 & $\mathbf{M}$ & 5 & Preop & $45 / 20 \quad 30$ & 15 & - & 2.7 & 890 & 440 & IV & & Starr $\mathbf{M}$ & - & - & $-\stackrel{\varphi}{\longrightarrow}$ \\
\hline .2 & $\mathbf{F}$ & 10 & Postop & $29 / 16 \quad 18$ & $\begin{array}{r}7 \\
24\end{array}$ & ? & $\begin{array}{l}3.7 \\
1.7\end{array}$ & $\begin{array}{r}390 \\
1510\end{array}$ & $\begin{array}{l}190 \\
380\end{array}$ & IV & - & Starr $\mathbf{M}$ & 94 & 49 & \\
\hline .2 & $\boldsymbol{F}$ & 10 & Postop & $32 / 12 \quad \frac{32}{24}$ & 15 & 6 & 3.2 & 600 & 22 & I-II & - & & 92 & AA & 54 \\
\hline .3 & $\mathbf{M}$ & 5 & Preop & $50 / 3542$ & 21 & 5 & $2 \cdot 0$ & 1680 & 840 & IV & & Starr $\mathbf{M}$ & - & 59 & $-\frac{1}{2}$ \\
\hline & & & Postop & $48 / 26 \overline{35}$ & 16 & 10 & $2 \cdot 8$ & 1000 & 110 & $\overline{T r}$ & + & An. T & - & & ( \\
\hline 14 & $\mathbf{M}$ & 14 & $\begin{array}{l}\text { Preop } \\
\text { Postop }\end{array}$ & $\begin{array}{ll}85 / 48 & 59 \\
70 / 38 & 50\end{array}$ & $\begin{array}{l}30 \\
19\end{array}$ & $\begin{array}{l}10 \\
10\end{array}$ & $\begin{array}{l}2.1 \\
2.3\end{array}$ & $\begin{array}{l}2250 \\
1740\end{array}$ & $\begin{array}{l}1100 \\
1080\end{array}$ & IV & + & $\begin{array}{l}\text { Starr M } \\
\text { An. T }\end{array}$ & $\begin{array}{l}197 \\
183\end{array}$ & $\begin{array}{l}55 \\
50\end{array}$ & \\
\hline
\end{tabular}


Table (cont) Haemodynamics and left ventricular function data

\begin{tabular}{|c|c|c|c|c|c|c|c|c|c|c|c|c|c|c|c|}
\hline $\begin{array}{l}\text { Case } \\
\text { No. }\end{array}$ & Sex & Age & & $P A$ & $P A W$ & $M G$ & $C I$ & $T P V R$ & $P A R$ & $N Y H A$ & $R M R$ & $\begin{array}{l}\text { Surgical } \\
\text { treatment }\end{array}$ & $L V E D$ & $E F$ & LVES \\
\hline 15 & $\mathbf{M}$ & 17 & Preop & $\begin{array}{l}65 / 25 \overline{39} \\
28 / 12 \frac{19}{16}\end{array}$ & 19 & 10 & $=$ & - & - & IV & & Starr M & 218 & 50 & 109 \\
\hline 16 & $\mathbf{M}$ & 10 & Preop & $82 / 35 \frac{10}{55}$ & 24 & 12 & 2.5 & $\overline{17} 60$ & 990 & $\begin{array}{l}\text { I-II } \\
\text { IV }\end{array}$ & - & $\begin{array}{l}\text { VSD clos. } \\
\text { Starr M }\end{array}$ & $\overline{159}$ & 58 & $\overline{67}$ \\
\hline & & & Postop & $38 / 13 \overline{23}$ & 14 & 8 & 3.6 & 510 & 200 & I & - & An. $T$ & 114 & 74 & 30 \\
\hline 17 & $\mathbf{F}$ & 11 & $\begin{array}{l}\text { Preop } \\
\text { Postop }\end{array}$ & $\begin{array}{l}46 / 26 \quad 34 \\
36 / 14\end{array}$ & $\begin{array}{l}14 \\
13\end{array}$ & $\overline{8}$ & $\begin{array}{l}2.5 \\
3.8\end{array}$ & $\begin{array}{r}1090 \\
530\end{array}$ & $\begin{array}{l}640 \\
253\end{array}$ & IV & - & Starr $\mathbf{M}$ & $\begin{array}{r}118 \\
87\end{array}$ & $\begin{array}{l}58 \\
62\end{array}$ & $\begin{array}{l}50 \\
33\end{array}$ \\
\hline 18 & $\mathbf{F}$ & 12 & Preop & $26 / 12 \frac{18}{18}$ & 9 & 7 & 3.8 & 380 & 190 & III & & Starr $M$ & - & - & - \\
\hline & $M$ & & Postop & $28 / 14 \overline{17}$ & 11 & $?$ & 4.8 & 280 & 100 & I-II & & & - & - & - \\
\hline 19 & $\mathbf{M}$ & 13 & $\begin{array}{l}\text { Preop } \\
\text { Postop }\end{array}$ & $\begin{array}{l}90 / 56 \quad 66 \\
24 / 8 \quad \sqrt{13}\end{array}$ & $\begin{array}{r}28 \\
6\end{array}$ & 20 & $\begin{array}{l}2.7 \\
3.6\end{array}$ & $\begin{array}{r}1960 \\
290\end{array}$ & $\begin{array}{r}1130 \\
150\end{array}$ & IV & - & Starr M & Z & 二 & 二 \\
\hline 20 & $\mathbf{F}$ & 14 & $\begin{array}{l}\text { Preop } \\
\text { Postop }\end{array}$ & $\begin{array}{l}85 / 40 \overline{60} \\
37 / 24 \overline{27}\end{array}$ & $\begin{array}{r}28 \\
9\end{array}$ & $\overline{8}$ & $\begin{array}{l}2.0 \\
3.8\end{array}$ & $\begin{array}{r}2400 \\
570\end{array}$ & $\begin{array}{r}1280 \\
380\end{array}$ & IV & & Starr $\mathbf{M}$ & - & - & - \\
\hline 21 & $\mathbf{M}$ & 15 & Preop & $84 / 4260$ & 20 & 20 & - & - & 2 & III & - & Starr $\mathbf{M}$ & 二 & - & - \\
\hline & & & Postop & $36 / 18 \quad 25$ & 11 & 8 & - & - & - & I & - & VSD clos. & - & - & - \\
\hline 22 & $\mathbf{M}$ & 10 & $\begin{array}{l}\text { Preop } \\
\text { Postop }\end{array}$ & $\begin{array}{l}96 / 53 \\
34 / 15\end{array}$ & 16 & - & $\begin{array}{l}3.9 \\
4.5\end{array}$ & $\begin{array}{r}1480 \\
430\end{array}$ & $\begin{array}{r}1150 \\
210\end{array}$ & IV & & Starr M & - & - & - \\
\hline 23 & $\mathbf{F}$ & - & $\begin{array}{l}\text { Preop } \\
\text { Postop }\end{array}$ & $\begin{array}{l}28 / 12 \frac{16}{16} \\
29 / 10 \frac{18}{18}\end{array}$ & 12 & $\frac{1}{3}$ & 二 & $=$ & $=$ & III & - & Starr $\mathbf{M}$ & 二 & - & - \\
\hline 24 & $\mathbf{F}$ & 7 & $\begin{array}{l}\text { Preop } \\
\text { Postop }\end{array}$ & $90 / 50 \frac{68}{68}$ & $\begin{array}{r}30 \\
6\end{array}$ & 24 & $\begin{array}{l}4.2 \\
5.9\end{array}$ & $\begin{array}{r}1300 \\
270\end{array}$ & $\begin{array}{l}720 \\
190\end{array}$ & IV & - & Starr $\mathbf{M}$ & $\overline{292}$ & $\overline{52}$ & $\overline{134}$ \\
\hline 25 & $\mathbf{M}$ & 11 & $\begin{array}{l}\text { Preop } \\
\text { Postop }\end{array}$ & $\begin{array}{l}37 / 16 \frac{24}{24} \\
26 / 10\end{array}$ & $\begin{array}{r}20 \\
9\end{array}$ & $\begin{array}{l}7 \\
7\end{array}$ & $\begin{array}{l}3.0 \\
3.4\end{array}$ & $\begin{array}{l}640 \\
330\end{array}$ & $\begin{array}{l}110 \\
120\end{array}$ & III & - & Starr $\mathbf{M}$ & $\begin{array}{r}80 \\
60\end{array}$ & $\begin{array}{l}56 \\
68 \\
70\end{array}$ & $\begin{array}{r}35 \\
115 \\
19\end{array}$ \\
\hline 26 & $\mathbf{F}$ & 11 & $\begin{array}{l}\text { Preop } \\
\text { Postop }\end{array}$ & $\begin{array}{l}45 / 20 \frac{30}{30} \\
34 / 16 \frac{10}{20}\end{array}$ & $\begin{array}{l}18 \\
12\end{array}$ & $\begin{array}{r}10 \\
6\end{array}$ & $\begin{array}{l}2.8 \\
4.2\end{array}$ & $\begin{array}{l}860 \\
380\end{array}$ & $\begin{array}{l}340 \\
150\end{array}$ & II & - & Starr $\mathbf{M}$ & $\begin{array}{r}181 \\
87\end{array}$ & $\begin{array}{l}68 \\
66\end{array}$ & $\begin{array}{l}17 \\
58 \\
30\end{array}$ \\
\hline 27 & $\mathbf{M}$ & 15 & $\begin{array}{l}\text { Preop } \\
\text { Postop }\end{array}$ & $\begin{array}{l}75 / 35 \overline{53} \\
54 / 19 \frac{\overline{3}}{33}\end{array}$ & $\begin{array}{l}37 \\
16\end{array}$ & $\begin{array}{l}28 \\
8.5\end{array}$ & $\begin{array}{l}2.9 \\
2.7\end{array}$ & $\begin{array}{r}1460 \\
980\end{array}$ & $\begin{array}{l}440 \\
500\end{array}$ & $\begin{array}{l}\text { IV } \\
\text { II }\end{array}$ & + & Hancock M & $\begin{array}{l}68 \\
82\end{array}$ & $\begin{array}{l}51 \\
52\end{array}$ & $\begin{array}{l}33 \\
40\end{array}$ \\
\hline 28 & $\mathbf{F}$ & 17 & $\begin{array}{l}\text { Preop } \\
\text { Postop }\end{array}$ & $\begin{array}{l}91 / 45 \frac{61}{29} \\
50 / 22 \frac{29}{50}\end{array}$ & $\begin{array}{l}47 \\
15\end{array}$ & $\begin{array}{r}40 \\
9\end{array}$ & $\begin{array}{l}1.5 \\
4.0\end{array}$ & $\begin{array}{r}3250 \\
580\end{array}$ & $\begin{array}{l}750 \\
280\end{array}$ & IV & - & $\begin{array}{l}\text { Starr M } \\
\text { An. T }\end{array}$ & $\begin{array}{r}242 \\
79\end{array}$ & $\begin{array}{l}41 \\
48\end{array}$ & $\begin{array}{r}143 \\
41\end{array}$ \\
\hline 29 & $\mathbf{M}$ & 11 & $\begin{array}{l}\text { Preop } \\
\text { Postop }\end{array}$ & $\begin{array}{l}73 / 44 \frac{58}{18} \\
28 / 14 \frac{18}{10}\end{array}$ & $\begin{array}{l}31 \\
14\end{array}$ & $\begin{array}{l}23 \\
13\end{array}$ & $\begin{array}{l}2.7 \\
5.3\end{array}$ & $\begin{array}{r}1720 \\
270\end{array}$ & $\begin{array}{r}800 \\
60\end{array}$ & IV & - & Hancock $M$ & 二 & $\begin{array}{l}76 \\
53\end{array}$ & 二 \\
\hline 30 & $\mathbf{F}$ & 6 & $\begin{array}{l}\text { Preop } \\
\text { Postop }\end{array}$ & $\begin{array}{l}61 / 33 \frac{48}{48} \\
32 / 15 \frac{20}{20}\end{array}$ & $\begin{array}{l}26 \\
15\end{array}$ & $?$ & $\begin{array}{l}2.7 \\
4.0\end{array}$ & $\begin{array}{r}1420 \\
400\end{array}$ & $\begin{array}{l}650 \\
100\end{array}$ & III & - & Starr M & $\begin{array}{r}135 \\
98\end{array}$ & $\begin{array}{l}49 \\
56\end{array}$ & $\begin{array}{l}69 \\
42\end{array}$ \\
\hline 31 & $\mathbf{F}$ & 11 & $\begin{array}{l}\text { Preop } \\
\text { Postop }\end{array}$ & $\begin{array}{l}42 / 31 \\
37 / 15 \quad \overline{36}\end{array}$ & $\overline{10}$ & $\bar{?}$ & $\begin{array}{l}2.3 \\
3.2\end{array}$ & $\begin{array}{r}1250 \\
630\end{array}$ & $\overline{380}$ & $\begin{array}{l}\text { IV } \\
\text { I-II }\end{array}$ & - & Starr $\mathbf{M}$ & 二 & 二 & - \\
\hline 32 & $\mathbf{M}$ & 13 & $\begin{array}{l}\text { Preop } \\
\text { Postop }\end{array}$ & $\begin{array}{l}70 / 35 \overline{50} \\
44 / 15 \overline{27}\end{array}$ & $\begin{array}{l}27 \\
12\end{array}$ & $\begin{array}{l}18 \\
?\end{array}$ & $\begin{array}{l}2.7 \\
3.2\end{array}$ & $\begin{array}{r}1480 \\
680\end{array}$ & $\begin{array}{l}680 \\
370\end{array}$ & $\begin{array}{l}\text { IV } \\
\text { I-II }\end{array}$ & - & Starr $\mathbf{M}$ & $\begin{array}{l}113 \\
108\end{array}$ & $\begin{array}{l}49 \\
61\end{array}$ & $\begin{array}{l}58 \\
42\end{array}$ \\
\hline 33 & $\mathbf{F}$ & 13 & $\begin{array}{l}\text { Preop } \\
\text { Postop }\end{array}$ & $\begin{array}{l}45 / 28 \overline{36} \\
24 / 10 \overline{15}\end{array}$ & $\begin{array}{r}23 \\
6\end{array}$ & $\begin{array}{l}8 \\
6\end{array}$ & $\begin{array}{l}3.3 \\
3.5\end{array}$ & $\begin{array}{l}870 \\
340\end{array}$ & $\begin{array}{l}320 \\
210\end{array}$ & III & - & Starr $\mathbf{M}$ & $\begin{array}{r}110 \\
87\end{array}$ & $\begin{array}{r}64 \\
.63\end{array}$ & $\begin{array}{l}40 \\
32\end{array}$ \\
\hline 34 & $\mathbf{M}$ & 14 & $\begin{array}{l}\text { Preop } \\
\text { Postop }\end{array}$ & $\begin{array}{l}60 / 33 \quad \frac{48}{31 / 11} \\
318\end{array}$ & $\begin{array}{r}30 \\
9\end{array}$ & $\begin{array}{r}10 \\
6\end{array}$ & $\begin{array}{l}2.5 \\
3.4\end{array}$ & $\begin{array}{r}1540 \\
420\end{array}$ & $\begin{array}{l}580 \\
210\end{array}$ & $\begin{array}{l}\text { III } \\
\text { I-II }\end{array}$ & - & $\begin{array}{l}\text { Starr M } \\
\text { Starr A }\end{array}$ & $\begin{array}{l}257 \\
129\end{array}$ & $\begin{array}{l}55 \\
68\end{array}$ & $\begin{array}{r}117 \\
40\end{array}$ \\
\hline 35 & $\mathbf{M}$ & 13 & $\begin{array}{l}\text { Preop } \\
\text { Postop }\end{array}$ & $\begin{array}{l}96 / 60 \\
34 / 12 \frac{73}{23}\end{array}$ & $\begin{array}{r}32 \\
5\end{array}$ & $\begin{array}{l}30 \\
4.5\end{array}$ & $\begin{array}{l}3.0 \\
3.6\end{array}$ & $\begin{array}{r}1950 \\
510\end{array}$ & $\begin{array}{r}1090 \\
400\end{array}$ & IV & - & Starr $\mathbf{M}$ & $\begin{array}{r}171 \\
55\end{array}$ & $\begin{array}{l}62 \\
57\end{array}$ & $\begin{array}{l}65 \\
23\end{array}$ \\
\hline 36 & $\mathbf{M}$ & 10 & $\begin{array}{l}\text { Preop } \\
\text { Postop }\end{array}$ & $\begin{array}{l}80 / 45 \quad 58 \\
41 / 25 \quad \underline{31}\end{array}$ & $\begin{array}{l}30 \\
14\end{array}$ & $\begin{array}{r}25 \\
7\end{array}$ & $\begin{array}{l}2.8 \\
3.5\end{array}$ & $\begin{array}{r}1660 \\
710\end{array}$ & $\begin{array}{l}800 \\
390\end{array}$ & $\begin{array}{l}\text { IV } \\
\text { II }\end{array}$ & + & Starr $\mathbf{M}$ & $\begin{array}{l}255 \\
250\end{array}$ & $\begin{array}{l}45 \\
47\end{array}$ & $\begin{array}{l}140 \\
133\end{array}$ \\
\hline 37 & $\mathbf{M}$ & 3 & $\begin{array}{l}\text { Preop } \\
\text { Postop }\end{array}$ & $\begin{array}{l}55 / 29 \\
28 / 13\end{array}$ & $\begin{array}{l}26 \\
12\end{array}$ & $\begin{array}{r}16 \\
5\end{array}$ & $\begin{array}{l}2 \cdot 0 \\
4.0\end{array}$ & $\begin{array}{r}1640 \\
400\end{array}$ & $\begin{array}{l}600 \\
160\end{array}$ & $\begin{array}{l}\text { IV } \\
\text { I-II }\end{array}$ & - & Starr $\mathbf{M}$ & - & E & - \\
\hline 38 & $\mathbf{M}$ & 16 & $\begin{array}{l}\text { Preop } \\
\text { Postop }\end{array}$ & $\frac{25}{42}$ & $\begin{array}{l}30 \\
12\end{array}$ & $\begin{array}{r}23 \\
7\end{array}$ & $\begin{array}{l}2.0 \\
3.2\end{array}$ & $\begin{array}{r}1800 \\
550\end{array}$ & $\begin{array}{l}600 \\
250\end{array}$ & IV & - & $\begin{array}{l}\text { Hancock M } \\
\text { An. T }\end{array}$ & $\begin{array}{l}82 \\
65\end{array}$ & $\begin{array}{l}43 \\
54\end{array}$ & $\begin{array}{l}47 \\
30\end{array}$ \\
\hline 39 & $\mathbf{M}$ & 15 & $\begin{array}{l}\text { Preop } \\
\text { Postop }\end{array}$ & 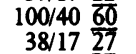 & $\begin{array}{l}24 \\
12\end{array}$ & $\frac{1}{8}$ & $\begin{array}{l}2 \cdot \\
4 .\end{array}$ & $\begin{array}{r}1850 \\
490\end{array}$ & $\begin{array}{r}1110 \\
270\end{array}$ & $\begin{array}{l}\text { IV } \\
\text { I-II }\end{array}$ & - & $\begin{array}{l}\text { Starr M } \\
\text { An. T }\end{array}$ & - & $\bar{z}$ & 二 \\
\hline 40 & $\mathbf{F}$ & 13 & $\begin{array}{l}\text { Preop } \\
\text { Postop }\end{array}$ & $\begin{array}{r}110 / 40 \quad \frac{60}{30 / 23} \\
33\end{array}$ & $\begin{array}{l}31 \\
16\end{array}$ & $\begin{array}{l}15 \\
10\end{array}$ & $2 \cdot 5$ & $\begin{array}{r}1850 \\
560\end{array}$ & $\begin{array}{l}890 \\
290\end{array}$ & IV & - & $\begin{array}{l}\text { Starr M } \\
\text { An. T }\end{array}$ & $\begin{array}{r}188 \\
63\end{array}$ & $\begin{array}{l}64 \\
73\end{array}$ & $\begin{array}{l}68 \\
17\end{array}$ \\
\hline 41 & $\mathbf{M}$ & 10 & $\begin{array}{l}\text { Preop } \\
\text { Postop }\end{array}$ & $\begin{array}{l}46 / 23 \\
37 / 18\end{array}$ & $\begin{array}{l}21 \\
15\end{array}$ & $\overline{9}$ & $\begin{array}{l}2.6 \\
3.5\end{array}$ & $\begin{array}{l}990 \\
570\end{array}$ & $\begin{array}{l}340 \\
230\end{array}$ & III & - & Hancock M & $\begin{array}{r}191 \\
84\end{array}$ & $\begin{array}{l}60 \\
40\end{array}$ & $\begin{array}{l}77 \\
51 \cdot\end{array}$ \\
\hline 42 & $\mathbf{F}$ & 10 & $\begin{array}{l}\text { Preop } \\
\text { Postop }\end{array}$ & $\begin{array}{l}30 / 17 \\
29 / 13\end{array} \frac{22}{17}$ & $\begin{array}{l}11 \\
13\end{array}$ & $\frac{-}{?}$ & $\begin{array}{l}3.6 \\
3.6\end{array}$ & $\begin{array}{l}480 \\
380\end{array}$ & $\begin{array}{r}240 \\
90\end{array}$ & II & - & $\begin{array}{l}\text { Starr M } \\
\text { Björk A }\end{array}$ & $=$ & $=$ & - \\
\hline 43 & $\mathbf{F}$ & 13 & $\begin{array}{l}\text { Preop } \\
\text { Postop }\end{array}$ & $\begin{array}{l}77 / 22 \\
28 / 12 \frac{\overline{44}}{19}\end{array}$ & $\begin{array}{l}26 \\
13\end{array}$ & $\frac{-}{5}$ & $\begin{array}{l}3.5 \\
5.0\end{array}$ & $\begin{array}{r}1010 \\
300\end{array}$ & $\begin{array}{l}410 \\
100\end{array}$ & $\begin{array}{l}\text { IV } \\
\text { I }\end{array}$ & - & Starr $\mathbf{M}$ & $\begin{array}{l}241 \\
114\end{array}$ & $\begin{array}{l}54 \\
55\end{array}$ & $\begin{array}{r}109 \\
51\end{array}$ \\
\hline 44 & $\mathbf{F}$ & 11 & $\begin{array}{l}\text { Preop } \\
\text { Postop }\end{array}$ & $\begin{array}{l}48 / 20 \\
28 / 10 \frac{30}{18}\end{array}$ & $\begin{array}{r}20 \\
7\end{array}$ & $\begin{array}{r}12 \\
6\end{array}$ & $2 \cdot 4$ & $\begin{array}{l}990 \\
380\end{array}$ & $\begin{array}{l}330 \\
180\end{array}$ & $\begin{array}{l}\text { IV } \\
\text { I }\end{array}$ & - & Starr $\mathbf{M}$ & $\begin{array}{r}200 \\
72\end{array}$ & $\begin{array}{l}55 \\
50\end{array}$ & $\begin{array}{l}89 \\
35\end{array}$ \\
\hline $\begin{array}{l}\text { Mean } \\
\text { Mean } \\
\text { p }\end{array}$ & & & $\begin{array}{l}\text { Preop } \\
\text { Postop }\end{array}$ & $\begin{array}{l}46.5 \\
23.4 \\
<0.0001\end{array}$ & $\begin{array}{l}25.6 \\
10.9 \\
<0.0001\end{array}$ & 二 & $\begin{array}{l}2.6 \\
3.8 \\
<0.0001\end{array}$ & $\begin{array}{l}1340 \\
533 \\
<0.0001\end{array}$ & $\begin{array}{l}590 \\
282 \\
<0.0001\end{array}$ & $\bar{z}$ & $\begin{array}{l}- \\
\overline{-}\end{array}$ & 二 & $\begin{array}{l}190 \\
103 \\
<0.0001 \\
\end{array}$ & $\begin{array}{l}57 \\
56 \\
>0.15 \\
\end{array}$ & - \\
\hline
\end{tabular}

A, aortic; An.T, tricuspid annuloplasty (De Vega); CI, cardiac index (1/min per $\left.\mathrm{m}^{2}\right)$; EF, ejection fraction (\%); LVED, left ventricular end-diastolic volume $\left(\mathrm{ml} / \mathrm{m}^{2}\right) ; \mathrm{LVES}$, left ventricular end-systolic volume $\left(\mathrm{ml} / \mathrm{m}^{2}\right) ; \mathrm{M}$, mitral; MG, mitral gradient (mmHg); NYHA, New York Heart Association grade; PA, pulmonary artery pressure ( $\mathrm{mmHg}$ ); PAR, pulmonary arteriolar resistance (dynes $\left.\mathrm{s} \mathrm{cm}^{-5} \mathrm{~m}^{-2}\right)$; PAW, pulmonary arterial wedge pressure (mmHg); RMR, residual mitral regurgitation; TPVR, total pulmonary vascular resistance (dynes $\mathrm{sm}^{-5} \mathrm{~m}^{-2}$ ); VSD clos., ventricular septal defect closure. 
Results

Complete data are shown in the Table.

PRESSURES

Pulmonary artery pressure was often much increased before operation, up to systemic level in some cases. The mean pulmonary artery pressure preoperatively ranged from 18 to 75 , mean $46.5 \mathrm{mmHg}$. After operation, there was a pronounced decrease to values ranging from 10 to 42 , mean $23.4 \mathrm{mmHg}$. This was highly significant $(p<0.0001)$. In five patients, however, there was mitral regurgitation postoperatively, and pulmonary arterial pressure remained high (above $\mathbf{3 0}$ mmHg) (Fig. 1a).

Mean pulmonary arterial wedge pressure was high before operation ( 9 to 45 , mean $25.6 \mathrm{mmHg}$ ); it decreased after operation ( 5 to 19 , mean $10.6 \mathrm{mmHg}$ ) $(p<0.0001)$. Five patients had pulmonary arterial wedge pressures higher than $15 \mathrm{mmHg}$ after operation; each had mitral regurgitation caused by paravalvar leak (Fig. 1b).

\section{CARDIAC INDEX}

The cardiac index rose from an average of 2.6 litres/ min per $\mathrm{m}^{2}$ before operation, to 3.8 litres/min per $\mathrm{m}^{2}$ $(p<0.0001)$ after operation (lower limit of normal for our laboratory is $3.0 \mathrm{l} / \mathrm{min}$ per $\mathrm{m}^{2}$ ). In patients with residual mitral regurgitation, cardiac index was lower than $3.5 \mathrm{litres} / \mathrm{min}$ per $\mathrm{m}^{2}$ postoperatively (Fig. 2).

\section{RESISTANCES}

Total pulmonary vascular resistance was moderately or much raised before operation (450 to 3150 , mean 1340 dynes $\mathrm{s} \mathrm{cm}^{-5} \mathrm{~m}^{-2}$ ), and it decreased after operation (250 to 970, mean 533) $(p<0.0001)$.

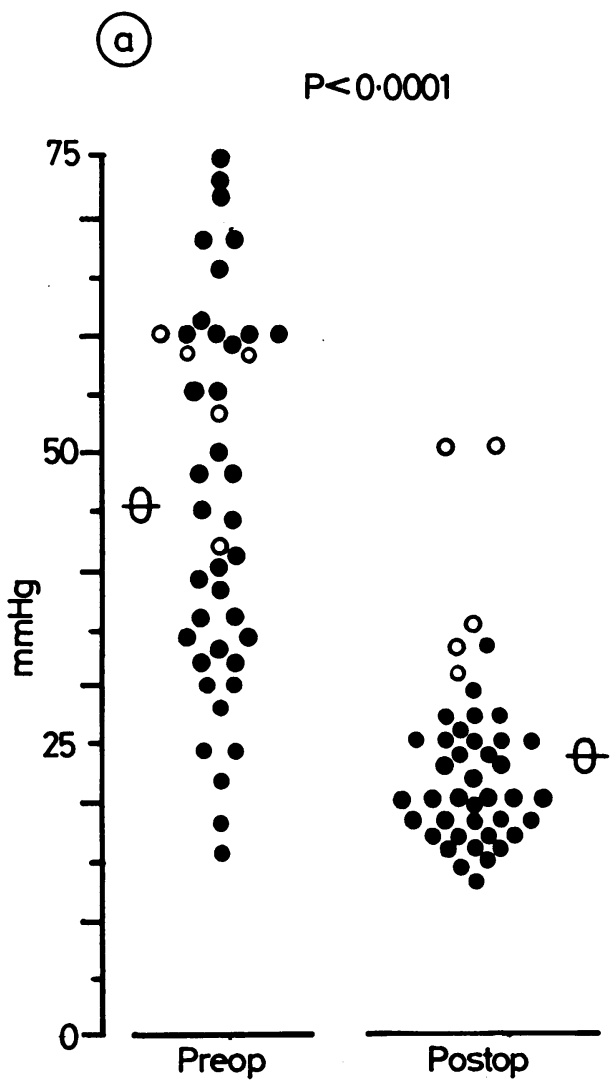

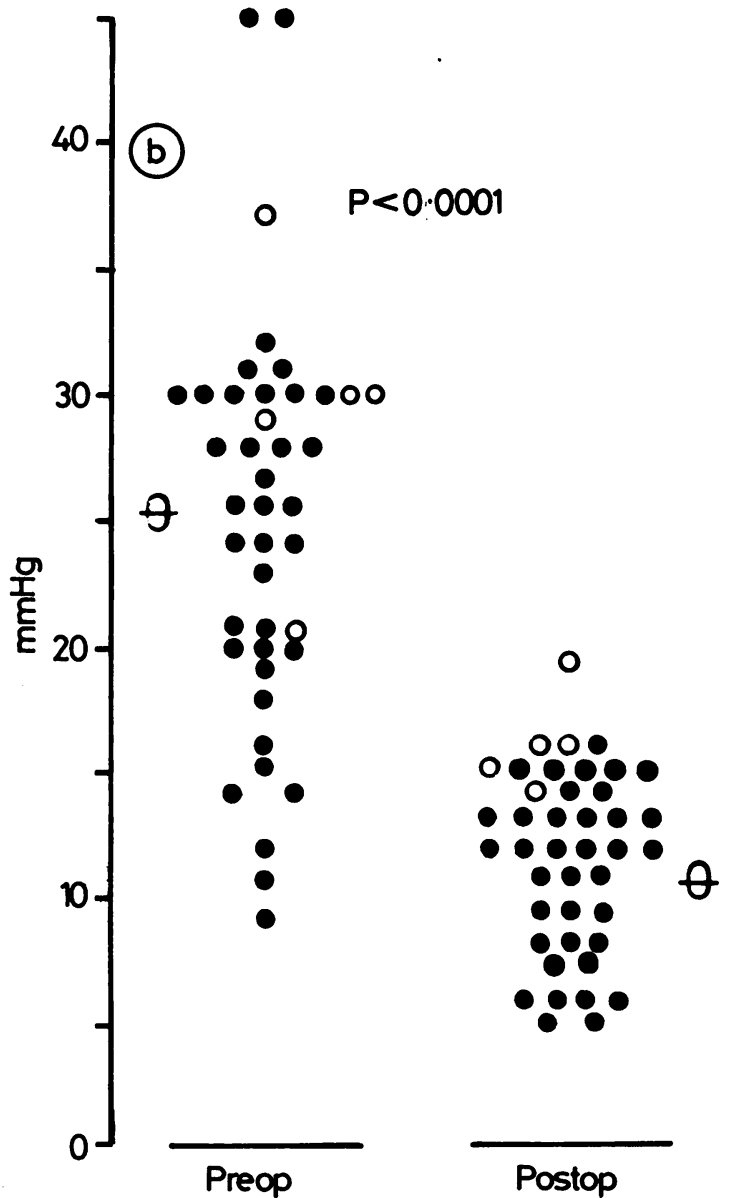

Fig. 1 (a) Mean pulmonary artery pressure before and after mitral valve replacement. Open circles indicate patients with residual mitral regurgitation (paravalvar leak). (b) Mean pulmonary wedge pressure before and after mitral valve replacement. Open circles indicated patients with residual mitral regurgitation. 


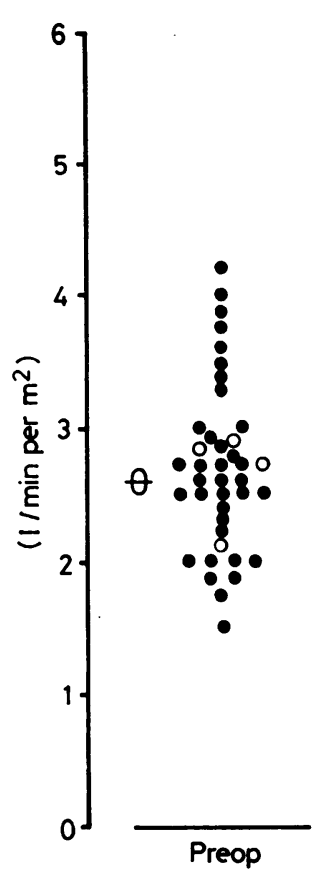

Fig. 2 Cardiac index before and after mitral valve replacement. Open circles indicate patients with residual mitral regungitation.

Pulmonary arteriolar resistance showed values between 120 and 1280, mean 590 dynes $\mathrm{s} \mathrm{cm}^{-5} \mathrm{~m}^{-2}$ before operation; these values fell to 60 to 430 , mean 282 dynes $\mathrm{s} \mathrm{cm}^{-5} \mathrm{~m}^{-2}$ after operation $(\mathrm{p}<0.0001)$ (Fig. 3). In all patients with preoperative values lower than 650 dynes $\mathrm{s} \mathrm{cm}^{-5} \mathrm{~m}^{-2}$, postoperative resistance was found to be normal ( $\leqslant 250$ dynes $\mathrm{s} \mathrm{cm}^{-5} \mathrm{~m}^{-2}$ ). If preoperative values exceeded 650 dynes $\mathrm{s} \mathrm{cm}^{-5} \mathrm{~m}^{-2}$, the pulmonary arteriolar resistance decreased, but a return to normal was not the rule. Patients with residual regurgitation had pulmonary arteriolar resistance above 500 dynes $\mathrm{s} \mathrm{cm}^{-5} \mathrm{~m}^{-2}$, unchanged from preoperative values (Fig. 3).

\section{RESIDUAL MITRAL GRADIENT}

A residual mitral gradient was commonly found after valve replacement in children. This was mild for the Starr-Edwards prostheses, with a gradient ranging from 2 to 10 , mean $6.9 \mathrm{mmHg}$. For the Hancock prostheses, the gradient was somewhat higher, between 6 and 13, mean $8.7 \mathrm{mmHg}$ (not significant). Though the difference between means is not statistically significant, it appears that one third of the patients with Starr-Edwards prostheses have insignificant gradients, between 2 and $6 \mathrm{mmHg}$; no child with a Hancock prosthesis had such a small gradient.

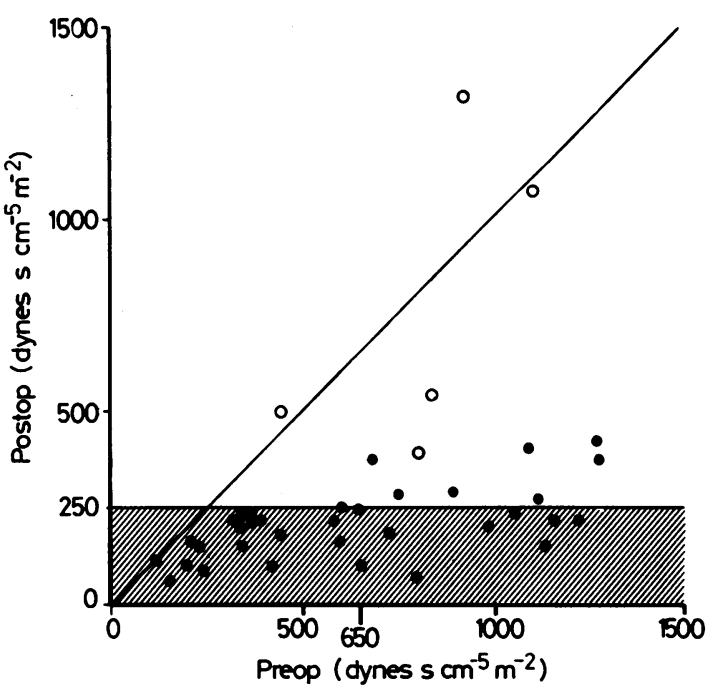

Fig. 3 Postoperative pulmonary arteriolar resistance (on the $y$ axis) plotted against preoperative resistance (on the $x$ axis), with the line of identity. Shaded area indicates normal values. Open circles are patients with residual mitral regurgitation. In cases without residual regurgitation, resistances always return to normal when the preoperative value does not exceed 650 dymes $\mathrm{s} \mathrm{cm-5} \mathrm{m}^{-2}$.

\section{VENTRICULAR VOLUMES}

End-diastolic left ventricular volumes were raised before operation ( 83 to 379 , mean $190 \mathrm{ml} / \mathrm{m}^{2}$ ). Seventeen patients had left ventricular end-diastolic volumes higher than $150 \mathrm{ml} / \mathrm{m}^{2}$, five patients had values between 110 and $150 \mathrm{ml} / \mathrm{m}^{2}$, but five had values lower than $100 \mathrm{ml} / \mathrm{m}^{2}$. Left ventricular end-diastolic volumes decreased after valve replacement and showed values between 55 and 250 , mean $103 \mathrm{ml} / \mathrm{m}^{2}$ $(p<0.0001)$ (Fig. 4). In 16 patients, the ventricular volume fell below $100 \mathrm{ml} / \mathrm{m}^{2}$, and six had values between 100 and $130 \mathrm{ml} / \mathrm{m}^{2}$. Five still had values above $130 \mathrm{ml} / \mathrm{m}^{2}$. Among these there was a case of moderate aortic regurgitation, who needed aortic valve replacement later; in three other patients, high postoperative left ventricular volume was related to residual mitral regurgitation.

\section{EJECTION FRACTION}

Left ventricular ejection fraction was measured before and after operation (Fig. 5). Before operation, about half of the patients had a depressed ejection fraction; the values ranged from 40 to $76 \%$, mean $57 \%$. Thirteen patients had values higher than $60 \%$, 10 patients had values between 50 and $60 \%$, and seven patients had values lower than $50 \%$. After operation, these values did not change significantly; they were 


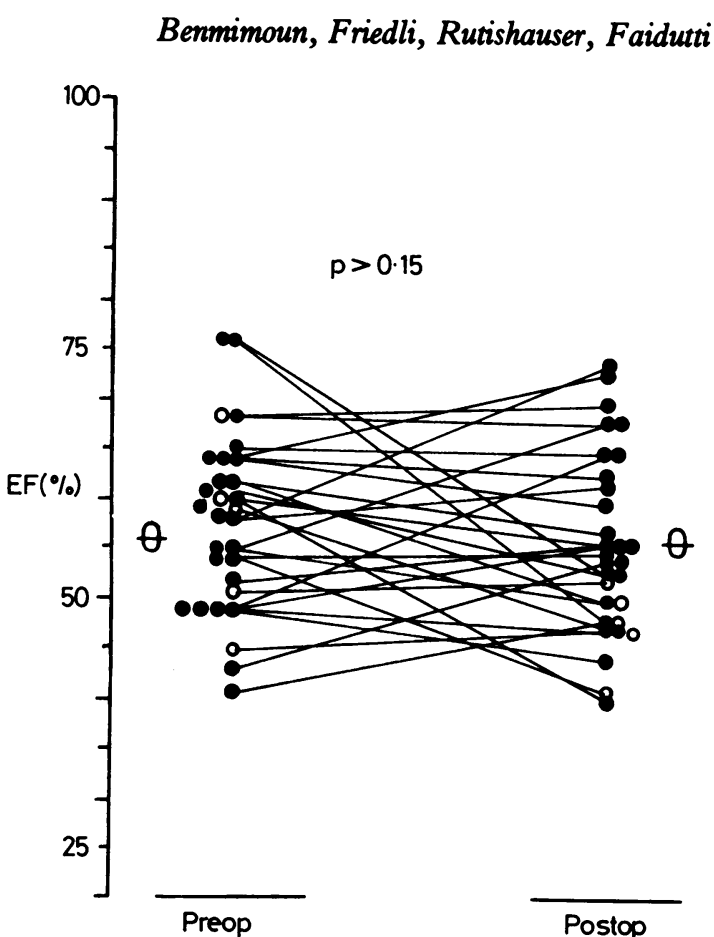

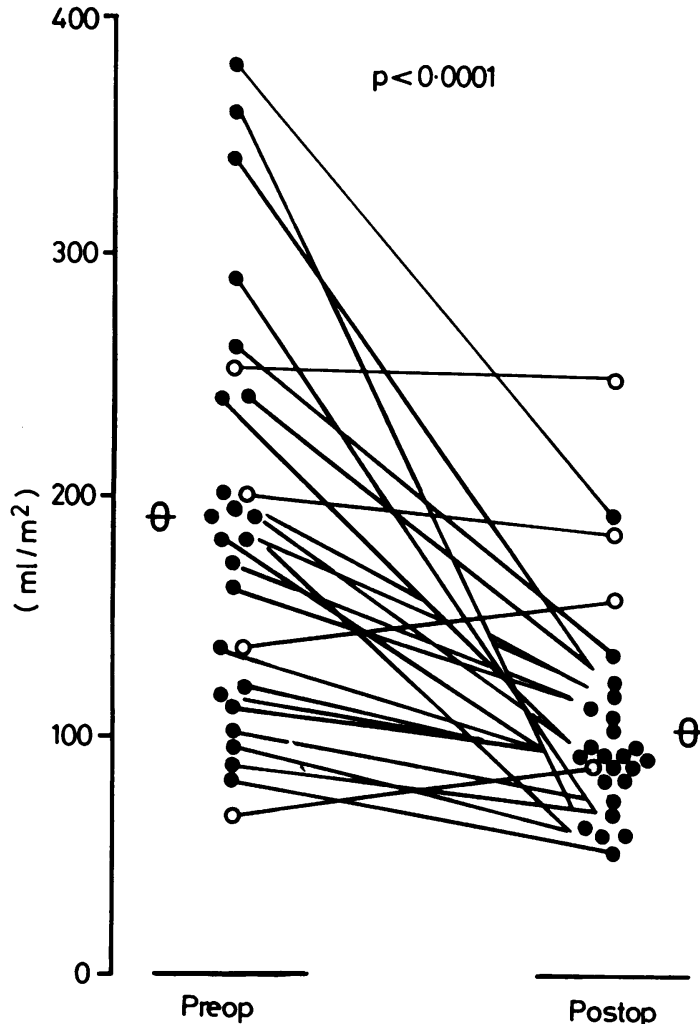

Fig. 4 Left ventricular end-diastolic volume (LVED) before and after mitral valve replacement. LVED decreased significantly after valve replacement in patients without residual mitral regurgitation. Open circles indicate patients with residual mitral regurgitation.

situated between 40 and $73 \%$, mean $56 \%$. No relation was found between the ejection fraction and age of the patient.

\section{Discussion}

Mitral valve replacement in children is usually restricted to patients who are severely symptomatic and in whom mitral valve repair is not feasible. The short term results are satisfactory and the children are much improved. ${ }^{7}$ Since valve replacement, however, is usually performed after a long period of progressive heart failure, complications are often present: longstanding left ventricular overload, together with sequelae of myocarditis in the case of rheumatic heart disease, may result in left ventricular dysfunction; on the other hand, chronically increased left atrial pressure will result in pulmonary hypertension. It is therefore interesting to assess postoperative haemodynamics and left ventricular function after valve replace-

Fig. 5 Left ventricular ejection fraction $(E F)$ measured before and after operation. The values are often below normal before as well as after operation, and there is no significant difference between preoperative and postoperative values.

ment and to compare the results with preoperative data.

HAEMODYNAMIC VARIABLES

Even extreme degrees of pulmonary hypertension and increased pulmonary vascular resistance secondary to mitral valve disease regress conspicuously in adult patients $^{3}{ }^{4}$ and children 6 who underwent adequate valve replacement, though a return to normal is not the rule. Thus, severe pulmonary hypertension is no longer a contraindication for operation, as had been suggested previously by some. ${ }^{10}$ This regression is partially the result of left atrial decompression: the pronounced reduction in left atrial pressure (pulmonary wedge pressure) explains the impressive reduction in total pulmonary vascular resistance. As shown in the present study, however, pulmonary arteriolar resistance, which is not directly related to left atrial pressure, also decreases conspicuously. The degree of increase in pulmonary arteriolar resistance before 
operation seems to be a determinant of residual postoperative pulmonary hypertension. Indeed, when preoperative values were below 650 dynes $\mathrm{s} \mathrm{cm}^{-5}$ $\mathrm{m}^{-2}$, a return to normal resistance was always observed. Above this level, some degree of pulmonary hypertension often persisted. In four of our seven patients with increased pulmonary arteriolar resistance after operation and no mitral regurgitation, the residual mitral gradient was somewhat above the average. In no case, however, did it exceed $10 \mathrm{mmHg}$ and we do not believe that this alone would explain the increased pulmonary arteriolar resistance. As the postoperative catheterisation took place fairly soon after operation, we do not know whether a further decrease may occur later in these cases. It is obvious, however, that the high resistances measured before operation are not the result of advanced pulmonary vascular disease, but rather of pulmonary arteriolar spasm. The situation is obviously different from pulmonary hypertension secondary to left to right shunt; indeed, pulmonary arteriolar resistance, measured two to four months after closure of a ventricular septal defect with pulmonary hypertension, does not change from preoperative values (B Friedli, unpublished data).

We have noted a significant increase in cardiac output after operation from the usually low preoperative values. It also appeared that some degree of residual mitral gradient may be found in children after mitral valve replacement: in our series, this was somewhat more significant for the Hancock porcine xenograft than for the Starr-Edwards prostheses. This is because for the same prosthetic valve diameter, the valve orifice is smaller in the Hancock prosthesis than in the Starr-Edwards prosthesis. As the postoperative study was done early, calcification of the porcine xenograft ${ }^{11}$ could not at that stage have been responsible for the relative stenosis.

Mitral regurgitation (paravalvar leak) was found in five patients. This relatively large number can be explained by the fact that the $\mathbf{4 4}$ patients catheterised in this study were selected from 171 children who had received valve prostheses during that period. Indications for recatheterisation were the presence of a residual murmur or preoperative pulmonary hypertension. It is obvious from the present study that, when significant residual mitral regurgitation is present, the haemodynamic variables remain abnormal, similar to the levels before operation.

\section{LEFT VENTRICULAR VOLUME AND FUNCTION}

To our knowledge, these variables have not been previously studied in children with valve prostheses. Left ventricular volume is considerably increased before operation, because of chronic left ventricular volume overload. Similar increases have previously been found experimentally ${ }^{12}$ as well as in adult patients. ${ }^{13}$
After operation, there is a pronounced decrease in volume, but a return to normal is not the rule: the mean left ventricular volume remains raised around $100 \mathrm{ml} / \mathrm{m}^{2}$. Left ventricular ejection fractions before operation vary over a wide range, but they are quite often abnormally low. There was no significant change in the mean, after operation, though some individual values did either increase or decrease. It must be emphasised that ejection fractions before and after mitral valve replacement cannot be directly compared because of the different haemodynamic setting. Indeed a series of variables, which determine left ventricular stroke volume, also influence ejection fraction. They are preload (left ventricular end-diastolic volume), afterload (left ventricular wall stress during ejection), myocardial contractility, and heart rate. In mitral regurgitation, preload is increased and afterload decreased. This should enhance ejection fraction; it has been shown that the "unloading" of the left ventricle in mitral regurgitation may mask the effect of depressed myocardial contractility.on overall pump function. ${ }^{14}$ This indicates that, in some of our patients, quite severe left ventricular dysfunction must have existed before operation, as the ejection fraction remains low in spite of the decreased afterload. One would also expect that ejection fraction decreases after mitral valve replacement, because of the increased afterload and decreased preload. This has been shown to occur in adults, ${ }^{15} 16$ but we did not find it in the present study. One explanation for this difference may be because left ventricular volume decreases considerably in children after valve replacement, more so than in adults. The reduction in left ventricular dimension (radius) reduces left ventricular stress, that is afterload. Thus, afterload increase after valve surgery in children may be less important than in adults.

As to the cause of the left ventricular dysfunction, before as well as after operation, two factors need to be considered: chronic volume overload may itself produce myocardial changes that are not completely reversible, as shown in the electron-microscopical studies of Papadimitriou et al. ${ }^{17}$ After closure of a ventricular septal defect-another cause of chronic left ventricular volume overload in childrenJarmakani et al. ${ }^{1}$ showed some left ventricular dysfunction, except in those patients who were operated on very early, before two years of life. In the present study, we have not been able to show a clear effect of age or duration of disease on left ventricular ejection fraction. There is obviously another possible cause of myocardial dysfunction in patients with rheumatic heart disease, that is myocardial fibrosis secondary to rheumatic myocarditis. Indeed, many children in this study have had repeated attacks of rheumatic fever.

The indication for valve replacement in children 
remains, as mentioned previously, the presence of severe, often longstanding heart failure. This is because of the complications of valve prostheses which occur in children, ${ }^{18} 19$ as well as in adults. ${ }^{20}$ The price of this conservative approach may be the presence of irreversible myocardial damage at the time of operation. In adults the results of valve replacement in grade IV heart failure are not as good as those in grade III heart failure. ${ }^{21}$ Should a new valve prosthesis with low thromboembolic risk and long durability be developed, valve replacement at an earlier stage, for example in grade II, would therefore be indicated.

\section{References}

1 Jarmakani JMM, Graham TP Jr, Canent RV Jr, Capp MP. The effect of corrective surgery on left heart volume and mass in children with ventricular septal defect. $A m \mathcal{F}$ Cardiol 1971; 27: 254-8.

2 Peterson CR, Herr R, Crisera RV, Starr A, Bristow JD, Griswold HE. The failure of hemodynamic improvement after valve replacement surgery. Ann Intern Med 1967; 66: 1-24.

3 Braunwald E, Braunwald NS, Ross J Jr, Morrow AG. Effects of mitral valve replacement on the pulmonary vascular dynamics of patients with pulmonary hypertension. $N$ Engl f Med 1965; 273: 509-14.

4 Zener JC, Hancock EW, Shumway NE, Harrison DC. Regression of extreme pulmonary hypertension after mitral valve surgery. Am f Cardiol 1972; 30: 820-6.

5 Bristow JD, Kremkau EL. Hemodynamic changes after valve replacement with Starr-Edwards prostheses. Am $\mathcal{F}$ Cardiol 1975; 35: 716-24.

6 Aryanpur I, Paydar M, Shakibi JG, Siassi B, Yazdanyar A. Regression of pulmonary hypertension after mitral valve surgery in children. Operative management of rheumatic mitral valve disease. Chest 1977; 71: 354-60.

7 Friedli B, Friedli GM, Ben Ismail M, Rouge JC, Hahn Ch, Faidutti B. Remplacement valvulaire chez l'enfant: résultats et suites éloignées chez 171 opérés. Schweiz Med Wochenschr 1981; 111: 1044-8.

8 Sandler H, Dodge HT. The use of single plane angiocardiograms for the calculation of left ventricular volume in man. Am Hear $\mathcal{F}$ 1968; 75: 325-34.

9 Carleton RA. Change in left ventricular volume during angiocardiography. Am f Cardiol 1971; 27: 460-3.
10 Barclay RS, Reid JM, Stevenson JG, Welsh TM, McSwan N. Long-term follow-up of mitral valve replacement with Starr-Edwards prosthesis. Br Heart $\mathcal{J}$ 1972; 34: 129-33.

11 Kutsche LM, Oyer P, Shumway N, Baum D. An important complication of Hancock mitral valve replacement in children. Circulation 1979; 60, suppl 1: 98-103.

12 McCullagh WH, Covell JW, Ross J Jr. Left ventricular dilatation and diastolic compliance changes during chronic volume overloading. Circulation 1972; 45: 94351.

13 Lewis BS, Gotsman MS. Left ventricular function during systole and diastole in mitral incompetence. $A m \mathcal{F}$ Cardiol 1974; 34: 635-43.

14 Eckberg DL, Gault JH, Bouchard RL, Karliner JS, Ross $\mathrm{J} \mathrm{Jr}$. Mechanics of left ventricular contraction in chronic severe mitral regurgitation. Circulation 1973; 47: 1252-9.

15 Schuler G, Peterson KL, Johnson A, et al. Temporal response of left ventricular performance to mitral valve surgery. Circulation 1979; 59: 1218-31.

16 Boucher CA, Bingham JB, Osbakken MD, et al. Early changes in left ventricular size and function after correction of left ventricular volume overload. Am $\mathcal{F}$ Cardiol 1981; 47: 991-1004.

17 Papadimitriou JM, Hopkins BE, Taylor RR. Regression of left ventricular dilatation and hypertrophy after removal of volume overload. Circ Res 1974; 35: 127-35.

18 Berry BE, Ritter DG, Wallace RB, McGoon DC, Danielson GK. Cardiac valve replacement in children. $\mathcal{F}$ Thorac Cardiovasc Surg 1974; 68: 705-10.

19 Mathews RA, Park SC, Neches WH, et al. Valve replacement in children and adolescents. $\mathcal{F}$ Thorac Cardiovasc Surg 1977; 73: 872-6.

20 Morrow AG, Newland-Oldham H, Elkins RC, Braunwald $E$. Prosthetic replacement of the mitral valve. Preoperative and postoperative clinical and hemodynamic assessments in 100 patients. Circulation 1967; 35: 962-79.

21 Najafi H, Dye WS, Javid H, Hunter JA, Ostermiller WE, Julian OC. Mitral valve replacement. Review of seven years' experience. Am $\mathcal{F}$ Cardiol 1969; 24: 386-92.

Requests for reprints to Dr B Friedli, Clinique de Pédiatrie, Hôpital Cantonal Universitaire, CH-1211 Geneva 4, Switzerland. 